\title{
El juicio Papon: analizar la mediatización de la experticia del historiador $^{1}$
}

\section{The Papon trial: analyzing the media coverage of historian's expertise}

Béatrice Fleury-Vilatte

Universidad Nancy II

beatrice.fleury-vilatte@univ-nancy2.fr

Jacques Walter

Universidad de Metz

walter@sha.univ-metz.fr

\begin{abstract}
7 Resumen
Durante el proceso Papon (1997-1998)² se citó a comparecer a historiadores como testigos expertos. La prensa generalista destacó positivamente la figura del historiador-experto debido a su esfuerzo por llevar a buen término la denuncia. Luego, en revistas "especializadas", surgió un fuerte desacuerdo en relación con la experticia del historiador. Para aclarar las causas y fundamentos de esta diferencia, se debe analizar uno de los tres lugares de pertinencia identificados por Patrick Charaudeau: el lugar de construcción del discurso. El conjunto confirma una evidencia olvidada por los estudios sobre la prensa: el tratamiento de un acontecimiento, aquí vinculado a la historia, depende de factores que van más allá del marco que lo asume en un período determinado.
\end{abstract}

Palabras clave: Juicio Papon, historiadores testigos-expertos, periodismo, lugares de pertinencia, sociología de las profesiones.

\section{Abstract}

During the Papon trial were cited to appear historians as expert witnesses. The general press highlighted the figure of the historian-expert positively due to his effort to carry out in good terms the claim. Then, in "specialized" journals, a deep disagreement arose regarding the

1. Traducido desde el francés por Jacqueline Fernández Cellier.

2. Maurice Papon, destacado político y hombre público francés, acusado en 1997 de colaborar con el régimen nazi; en particular, se le imputó la deportación de más de mil quinientos judíos, con la consecuencia de su posterior exterminio (N. del E.). 
historian expertise. To clarify the causes and bases of this difference, we must analyse one of three relevance points identified by Patrick Charaudeau: the discourse construction point. The set confirms a rather forgotten evidence by the press studies: the treatment of an event, here linked to History, depending of factors beyond the framework wich assumes in a given period.

Keywords: Papon trial, historians, journalism, points of relevance, sociology of the professions.

\section{Introducción}

Para el analista del discurso, los fenómenos que tienen relación con la mediatización son doblemente interesantes: ellos enseñan sobre la relación de los periodistas con su entorno particular y aclaran ciertos aspectos del funcionamiento mediático en el cual trabajan grupos profesionales.Así,el discurso dela información produce un complejo ajuste entre informaciones relacionadas con apuestas sociales y parámetros contextuales vinculados con la actividad periodística y editorial. Si bien el enunciado y las representaciones de los acontecimientos alcanzan las preocupaciones de una sociedad, más que revelar la opinión del momento, muestran la relación de los medios con la sociedad según una objetivación del rol que, de forma implícita, estos últimos se atribuirían o que les sería atribuido. Esto es particularmente evidente en el caso de un hecho histórico y judicial problemático -en este caso, relacionado con la Segunda Guerra Mundial- más tendiente a favorecer tomas de posición. Los periodistas se muestran aquí como importantes actores del debate público, privilegiando y publicitando ciertas interpretaciones de este hecho en perjuicio de otras. Así, producen justificaciones de sus dichos (Charadeau, 2001: 9), no solamente para dar a conocer una verdad de los hechos, sino -en un registro más cercano al campo político que al campo de la información- para persuadir, o influir.

Estos elementos se encuentran en el tratamiento dado por dos tipos de prensa generalista y especializada - al juicio de Maurice Papon que tuvo lugar en Bordeaux entre el 7 de octubre de 1997 y el 2 de abril de 1998. El antiguo secretario general de la prefectura de Gironda, quien hasta entonces había tenido una bella carrera política, es acusado de haber colaborado con la organización de la deportación de 1560 judíos de la región de Bordeaux hacia el campo de Drancy, cuyo destino final fue Auschwitz. Tal como sucedió en el caso del proceso del nazi Klaus Barbie (1987) y contrariamente a lo que sucedió con el miembro de la milicia Paul Touvier (1994), un poco dejado de lado por los periodistas (Conan y Rousso, 1994: 254), las publicaciones informaron ampliamente de las audiencias, enviando periodistas al lugar mientras duró el período de sesiones del Tribunal Penal. ¿Por qué este interés ? Después del proceso Touvier, era la segunda vez que se realizaba un proceso por complicidad de crímenes contra la humanidad, en virtud del carácter imprescriptible de este tipo de crímenes, instaurado en Francia por la ley No 64-1326 del 26 de diciembre de 1964. 
Considerando que esta acusación estaba desfasada en relación con los "actos justiciables que se inscriben en la definición jurisprudencial del crimen contra la humanidad" (Ribémont, 2001:76), seinicióuna reflexión sobrela relación entre justicia, testimonioymemoria. Esbozada en la prensa generalista, fue ampliamente desarrollada en la prensa especializada. Uno de los puntos centrales del debate era la evaluación del impacto del carácter de imprescriptibilidad que condujo a algunos a considerar que el testigo no solamente podía ser aquél que relatara hechos con los cuales hubiese tenido relación directa: "Instaurando una temporalidad inmóvil, el régimen de imprescriptibilidad nos vuelve a todos contemporáneos de los crímenes pasados cuyos culpables aún están vivos" (ibid.: 78). De hecho, los historiadores ${ }^{3}$ se transformaron en testigos completamente indicados y totalmente legítimos en lo que Jean-Paul Jean y Denis Salas (2002) llaman "procesos para la memoria". Citados a comparecer como testigos-expertos, fueron cuestionados por sus pares, entre ellos Henry Rousso, director del Instituto de Historia del Tiempo Presente (IHTP). Este tema, al igual que la condena de Maurice Papon a diez años de reclusión criminal y a la pérdida de los derechos cívicos, se manifestó de dos formas cuyo análisis pone en evidencia una configuración contrastada del funcionamiento de los medios. Un primer estudio (Fleury-Vilatte y Walter, 2004) permitió demostrar cómo soportes generalistas estuvieron unidos -sin estrategia programada- mediante una lógica de acuerdo, en el tratamiento de estos dos temas, mientras que en soportes de la prensa especializada -aparecidos posteriormente y analizados en un segundo estudio (Fleury-Vilatte y Walter, 2003) - surgieron desacuerdos. Así, los medios de gran difusión aparecieron como pantallas, en el sentido que se proyectaban en ellos las sombras del desacuerdo. Por su parte, los soportes especializados revelaron lo que esas sombras disimulaban, permitiendo el acceso a un espacio impensado cuya explicación aclara de otro modo las prácticas de la prensa generalista.

Situado en el campo de las ciencias de la información y de la comunicación, el tema era dilucidar las causas y fundamentos de esta diferencia, a partir del análisis de uno de los tres lugares de pertinencia identificados por Patrick Charaudeau (1997): el lugar de construcción del discurso. Considerando que la comprensión de los procesos en curso en ese lugar sólo puede hacerse considerando los otros dos lugares -lugar de las condiciones de producción y

3. Concretamente, Jean-Pierre Azéma (profesor universitario en el Instituto de Estudios Políticos de París), Marc Olivier Baruch (investigador del Instituto de Historia del Tiempo Presente/CNRS) y Robert O. Paxton (profesor de la Universidad de Columbia en Nueva York) fueron citados a comparecer por el Ministerio Público. Por su parte, Henri Amouroux (periodista, decano de la sección de historia y geografía de la Academia de Ciencias Morales y Políticas), Michel Bergès (profesor universitario en ciencias políticas) Jacques Delarue (comisario de división honorario, historiador), André Gouron (profesor universitario en derecho), André Kaspi (profesor universitario, presidente de la Comisión Francesa de los Archivos Judíos), Jean Lacouture (periodista), Maurice Rajfus (historiador y militante de la extrema izquierda), Henry Rousso (director de investigación en el CNRS, director del Instituto de Historia del Tiempo Presente) fueron citados por la defensa; Philippe Burrin (profesor en el Instituto de Altos Estudios Internacionales de Ginebra), Eberhard Jäckel (profesor de la Universidad de Stuttgart), René Rémond (profesor universitario jubilado, presidente de la Fundación Nacional de Ciencias Políticas) Pierre Vidal-Naquet (historiador, director de estudios en la Escuela de Altos Estudios y Ciencias Sociales), fueron citados por las partes civiles. 
lugar de las modalidades de interpretación durante la recepción-, hemos recurrido a la noción de escena que permitió poner en evidencia la dinámica de los intercambios entre los espacios de sentido. Para esto, asociando al análisis de discurso teorías tomadas de la sociología (sociología de la experticia, sociología de las profesiones provenientes de la corriente interaccionista) y de la historia (historiografía, historia del tiempo presente) -teorías bastante poco utilizadas en este tipo de investigación - fueron seriados los modos de articulación y de complejidad entre cuatro escenarios de experticia implicados en el asunto (judicial, mediática, histórica, política), sabiendo que el escenario mediático tiene la capacidad de abarcar los otros escenarios e influir en el proceso de conocimiento de una sociedad. Por lo tanto, en una primera parte, se presentarán los resultados relacionados con el análisis contrastivo de los dos corpus; en las dos siguientes, se tratarán los problemas planteados por cada fase, según las teorías citadas. El conjunto confirmará una especie de evidencia, pero muy poco considerada en los estudios sobre la prensa: el tratamiento de un acontecimiento, vinculado aquí con la historia, depende de factores que van más allá del soporte que lo asume durante un período determinado.

\section{El proceso: una doble temporalidad mediática}

Para evaluar el efecto real del proceso de Bordeaux, en especial en lo que se refiere a la relación específica que lo asocia con los medios, hay que remitirse al inicio de los años 80 cuando los periodistas Claude Angeli y Nicolas Brimo se reúnen con Michel Slitinsky, quien había sido detenido en 1942 con su hermana por inspectores de la policía francesa en Bordeaux: deseaba iniciar seguimientos contra Maurice Papon, ya que había sido informado sobre las actividades de éste por un investigador de Bordeaux (Michel Bergès). En mayo de 1981, este contacto dio origen a un primer artículo en la revista semanal Le Canard enchainé, que fue el punto de partida del asunto. A partir de ese momento el caso volvió regularmente a la esfera pública acentuándose en el período preparatorio del proceso, luego durante su desarrollo. Después del veredicto, se publicaron libros sobre el tema e informes preparados por la prensa especializada. Con el fin de estudiar los particularismos de cada una de las fases y de cada uno de los lugares del debate, se confrontaron dos corpus: el primero, que se extendió desde el mes de octubre de 1997 hasta el mes de enero de 2001, estaba compuesto por cuatro periódicos (L'Humanité, Le Figaro, Le Monde, Libération) y cuatro revistas (L'Événement du jeudi, L'Express, Le Nouvel Observateur, Tééréma); el segundo incluía números de la prensa especializada: Autrement (Jean y Salas, 2002), Droitet société (1998), L’Histoire (1998), Le Débat (1998). Su análisis se completó con la inclusión de obras de historiadores o periodistas, tales como Henry Rousso y Philippe Petit (1998a), Éric Conan (1998), Jean-Michel Dumay (1998) y mediante entrevistas con Henry Rousso, opuesto a la participación de los historiadores, y Marc Olivier Baruch, quien fue testigo.

Varios hechos sobresalientes se desprenden del análisis de estos documentos. Primero, se evoca a menudo una sobremediatización que influye en la serenidad de los debates y puede llegar a influir en el veredicto; sin embargo, no es la mediatización la que provocó las mayores 
discusiones, a diferencia de las relaciones entre magistrados e historiadores y del estatus de cada uno en este proceso que es tanto el juicio de un hombre como el de un régimen. Entre los protagonistas, los historiadores ocuparon un lugar importante: se volvió a hablar sobre puntos particulares del período de Vichy o del desarrollo de las audiencias, en fechas cercanas a las de su presencia en el tribunal, haciendo así visible la competencia profesional que los autorizaba a pronunciarse frente a la justicia. Otros, que se negaron a testificar, comentaron el estatus de testigo-experto del historiador y los contenidos de la experticia, criticando a veces el rol demasiado importante de los medios.

Luego, el análisis de la puesta en escena de la figura del historiador testigo-experto permitió la actualización de los procedimientos mediante los cuales la prensa generalista -que contribuyó durante años a sacar adelante la demanda y que ambicionaba también una función pedagógica respecto al período, en nombre del deber de memoria- minimizó lo que habría podido perjudicar la precisión del proceso, callando especialmente los desacuerdos dentro de la comunidad de historiadores. También permitió comprender cómo la prensa protegió o amplificó una representación dominante del período de Vichy, representación de la cual era parte interesada y que estaba de acuerdo con la expectativa de un veredicto que condenara al acusado e hiciera justicia a las víctimas. De estas disposiciones surge la construcción de la figura del experto legítimo que se encuentra casi sin distinción en los diversos títulos. Esta figura fue derribada en las revistas especializadas, mediante un claro movimiento de aumento de la generalidad en los temas abordados y en los participantes invitados. Por ejemplo, será el caso de los procesos de Klaus Barbie y Paul Touvier, o de los procesos por crímenes contra la humanidad, en que también intervendrán historiadores cuyo campo de predilección no es el tiempo presente, o incluso juristas, filósofos, políticos que teorizan la relación entre justicia e historia.

Finalmente, esta vez desde una vertiente teórica, el análisis de estas dos prensas - diferentes en el funcionamiento y la postura adoptada durante el debate- se basa en dos recursos diferenciados. Para dilucidar los términos de la práctica que aparece en la prensa generalista, el recurso de la sociología de la experticia y principalmente la noción de escenario de experticia reveló ser fecundo para pensar la articulación de los lugares, actores y tipos de palabras, sabiendo que los escenarios interactúan y se regulan unos a otros, induciendo de este modo una cierta vaguedad en cuanto al estatus y al discurso de los participantes. Para estudiar las revistas especializadas, más específicamente relacionadas con la visión que los historiadores tenían de sí mismos, es la sociología de las profesiones y, en este marco, la noción de "segmento" (Bucher y Strauss, 1961) la que permitió desenredar la compleja madeja de un campo en mutación.

\section{La prensa generalista: escenarios de experticia en interrelación}

De alguna manera, la sociología de la experticia fue impuesta por el objeto. Además, fue utilizada por investigadores que participaban en el debate historiográfico (e.g. Rousso, 1998; 
Damamme y Lavabre, 2000; Rousso, 2002) y fue desarrollada incluso más frontalmente en el artículo de la revista Cahiers politiques, donde Thomas Ribémont (2001) mostraba lo que pasaba durante este proceso en todos los dispositivos de experticia para saber si el testimonio de los historiadores respondía a esta categoría. Por lo tanto, para especificar nuestro aporte, es necesario aclarar la forma en que consideramos la experticia.

Sobre la base de ciertos trabajos, en particular los de Jean-Yves Trépos $(1996,2002)$, primero hay que diferenciar al experto del profesional que interviene en el marco de una actividad rutinaria. En efecto, "el experto actúa puntualmente gracias a competencias específicas, con la misión de formular un juicio u ofrecer una solución, en una situación problemática" y "articula: un saber hacer, es decir un repertorio de claves de acción; un saber comprender, es decir un modo de apreciar hasta qué punto hay que movilizar los saber hacer; un saber combinar, es decir una estrategia para disponer las diferentes fases de su intervención" (Trépos, 2002: 8). Tantas capacidades que efectivamente es posible encontrar en artículos de la prensa generalista y luego se pueden discutir en revistas especializadas. Sin embargo, si bien el tema del experto estuvo al centro del proceso, se manifestó en forma contrastada en los cuatro escenarios presentes, siendo el escenario mediático el que ocupó el lugar central en esta configuración, ya que tal como lo hacen notar Valérie Amiraux y Daniel Cefaï (2002), "el experto circula entre varios escenarios institucionales [... Él participa en varios espaciostiempos de reflexión, de polémica, de conflictos con otros protagonistas. Su credibilidad está reforzada por la circulación de sí mismo y de su saber entre los diferentes escenarios -teniendo el reconocimiento de los medios un rol primordial para forjar una reputación".

Se desprende del análisis del discurso que la prensa se mostró dinámica en la valorización o en la atenuación de ciertas interpretaciones de la situación jurídico-histórica. Por ejemplo, si bien abordó el "dilema de los historiadores citados a comparecer" (Le Monde, 16.10.97), no se discutieron las razones: éstas aparecían en los correos de rechazo dirigidos al juez o en entrevistas con los historiadores involucrados, donde ellos analizaban diversos problemas relacionados con el período de Vichy. Por cierto, puede parecer sorprendente que ciertos historiadores que se negaron a comparecer hayan aceptado responder a los periodistas; Henry Rousso lo justificó diciendo que prefería el escenario mediático al escenario judicial para hacer escuchar su voz y entregar una experticia sin estar obligado a formular un juicio al cual habría seguido una decisión. Sin embargo, este último fue discreto en el escenario mediático. Esta discreción fue una elección personal, pero también el resultado de una lógica implícita de parte de ciertas publicaciones, ya que la opinión del historiador no correspondía a la figura tranquilizadora que daban de la experticia. Del mismo modo, la palabra de los historiadores jamás fue cuestionada, no más de lo que fue discutida su importancia, bastando su rol de testigo-experto para avalarla.

¿Por qué tal reserva? Para comprenderlo, es necesario reubicar el acontecimiento en el contexto más amplio de las relaciones entre historiadores y periodistas. Desde los años 80, Vichy fue objeto de una atención específica debido a procesos como los de Klaus Barbie o de 
Paul Touvier, procedimientos de reparación o demandas interpuestas por las víctimas, para los cuales se realizaron pruebas periciales. Los historiadores que trabajaban en este período se encontraron entonces en el centro de las preocupaciones sociales. Sus producciones son conocidas por ciertos periodistas que, en los temas que tienen relación con la historia contemporánea y sobre todo con la Shoah -elemento central del acta de acusación-dan prueba de gran competencia. Durante el proceso, sus firmas figuraron al lado de las de los cronistas judiciales: Nicolas Weill para el diario Le Monde (ya había escrito con Annette Wieviorka, 1994) y Éric Conan para la revista L'Express, siendo este último también uno de los redactores jefe de la revista Esprit (y colaborador de Henry Rousso, 1994). Esta particularidad hizo del escenario mediático un espacio de palabra experta cuyos actores no solamente restituyeron el saber historiador, sino que lo co-construyeron.

Se desprende de estos elementos, la forma en que destaca la prensa a ciertos historiadores entre los cuales figuraba Marc Olivier Baruch, quien, siendo desconocido por los medios antes del proceso, fue imprescindible durante él. El recorrido de este último no es ajeno a la mediatización de la cual fue objeto. Historiador de la administración -entre las cuales: Vichy, una extraña especialidad que contribuye a desarrollar (Baruch, 1997)-,investigador en elIHTP, también fue alto funcionario de la administración francesa durante seis años. En los periódicos, siempre se precisó su calidad de egresado de la Ecole Polytechnique y de la Ecole Nationale d'Administration. Parecían garantizar el testimonio-experto requerido, lo que constituye una observación clásica en sociología de la experticia. En consecuencia, la mayoría de las informaciones que los periodistas le solicitaron se referían a la capacidad de independencia de un alto funcionario de la Francia de Pétain. Por esto y por los numerosos artículos relacionados con este historiador, la prensa, preocupada -en su conjunto- por encontrar los argumentos decisivos que permitieran condenar a Maurice Papon, instrumentalizó las opiniones emitidas por dicho historiador. Si bien esta forma de actuar fue emblemática de las representaciones de la experticia historiadora en la prensa generalista, se distinguió de la de las revistas especializadas cuyo análisis sobresalió de las diferentes representaciones, tanto por los contenidos como por las posturas implicadas. El estudio contrastivo permitió entonces comprender los fundamentos y las causas de esta diferencia así como sus manifestaciones en los escenarios de experticia.

\section{Las revistas: un campo profesional en transformación}

Lo hemos visto: la prensa es un espacio de manifestación de la competencia profesional y de la experticia. Sin embargo, en este tipo de sociología, no debiera olvidarse su modo de construcción y las diferencias en la definición de "buenos" modelos (Walter, 1995). Lo que quiere decir que la construcción es también un enfrentamiento en un campo y que la estabilización de un modelo nunca es definitiva. Por esto se quiere insistir en el hecho que un medio profesional, compuesto -entre otros elementos- por especialidades, está atravesado por conflictos entre lo que se llama, luego de Rue Bucher y Anselm L. Strauss (1961: 82 y ss.), segmentos. Esta palabra designa a grupos de personas que comparten una concepción 
cercana de su actividad. De hecho, la gran mediatización del proceso y de ciertos historiadores cristalizó oposiciones entre segmentos (e.g. sobre el rol del especialista que puede querer, o no, separar las posturas de investigador, de experto, de pensador; vid. Heinich, 2002). Estas oposiciones se podían leer entre líneas en el tratamiento que daba la prensa al proceso; salieron a plena luz en la prensa especializada, alimentadas por las tomas de posición de especialistas o actores del acontecimiento.

Del mismo modo, el tema consistió en relacionar los elementos de una polifonía interpretativa, con las intervenciones de los historiadores del "momento Papon", con el fin de analizar los efectos epistemológicos de la actualidad en la historia. Mediante el uso de esta expresión, quisimos decir que, en la historiografía del período de Vichy, el proceso constituía ciertamente el apogeo de la "segunda depuración", según la feliz fórmula de Henry Rousso, pero también el momento de hacer visible procesos de construcción y de derribamiento de lo que sería la experticia -judicial o no- en el período considerado. Finalmente, este proyecto se inscribe en una proposición de naturaleza metodológica que sugiere analizar el discurso de los medios, cuando se trata de testimonio, según: el nivel de las polémicas entre expertos, el de los dispositivos mediáticos y el de los factores históricos (Walter, 2003). Estos niveles están necesariamente presentes en forma simultánea en cada uno de los puntos de controversia, incluso si se nota un énfasis de uno u otro según la naturaleza de los desacuerdos.

Sin lugar a dudas, deben considerarse varios parámetros para analizar la articulación de las competencias profesionales y el marco de ejercicio de éstas. Uno concierne al procedimiento utilizado en los procesos por crímenes o complicidad de crímenes contra la humanidad, que ubica a

los jueces y a los historiadores en un mismo espacio real y simbólico, volviéndolos, en niveles diferentes, actores de un mismo escenario. Se encuentran en competición, por no decir en competencia, cada uno haciendo interpretaciones sobre el pasado que pueden ejercer una gran influencia en el espacio público (Rousso, 2002: 61).

Otro corresponde al terreno en sí -el tiempo presente- que implica una dificultad para los investigadores de establecer distancia con el objeto de sus investigaciones. A falta de ésta, corren el riesgo de no distinguirse de los otros campos profesionales, entre los cuales se encuentra el periodismo. Entonces, está en tela de juicio la legitimidad de una especialidad en el seno de los enfoques historiadores. El paso de las fronteras de un mundo (la investigación) a otros (el judicial, el político), sustituido incluso por otro (el mediático), deja ver desacuerdos internos, susceptibles de debilitar la subdisciplina. En fin, es un tema que tiene más repercusiones al exterior del grupo: el lugar del judeocidio en la interpretación de la historia de Vichy.

A este respecto, la interpretación de Henry Rousso es esencial en el dispositivo, ya que se opone a la de los periodistas, entre los cuales Nicolas Weill. Según el historiador, la centralidad del judeocidio en la memoria de este período tiene como principal consecuencia una disminución de las responsabilidades del ocupante alemán, una homogeneización de lo que fue 
la Resistencia, una vacilación sobre la legitimidad de las instancias que debía encarar Francia, una redefinición de los criterios que permiten evaluar el comportamiento de los franceses. No solamente ve un hiato entre la realidad de la época y la memoria actual, sino que estima que esta distorsión resulta de la disposición de las jóvenes generaciones para denunciar a Vichy y su antisemitismo para refundar una "identidad nacional". Presente en otras contribuciones (e.g. Thibaud, 1997), este análisis es lo que el historiador Philippe Burrin (1998) califica de versión "fundamentalista"; una versión incluso defendida por Nicolas Weill en el diario Le Monde, que muestra a Vichy como "co-autor" del Genocidio. Finalmente, el debate demostró que, discutiendo, los historiadores tendieron a proteger su campo de competencias profesionales, mientras que el periodista de Le Monde alentaba una vertiente de los historiadores por y para la cual el conocimiento debe honrar un compromiso en el espacio público. Philippe Burrin (1998) señalaba también que, sin ser nombrados, su colega Jean-Pierre Azéma y él mismo habían sido acusados por Nicolas Weill (Le Monde, 03.04.98) de ser de aquellos que proponen "sutilezas con virtudes dispensadoras", cuando incluso la mayoría de los historiadores especialistas del período- están de acuerdo con este análisis. Él veía en esto una concepción de la historia, marcada por la preocupación de escribirla "según los efectos que (ella) puede tener en la memoria de una época tal como quisiéramos verla fijada actualmente”. Durante el proceso de Bordeaux, y sin que se haya tomado distancia de ella, esta dimensión estructuró el discurso de la prensa generalista. Al contrario, en la prensa especializada, particularmente en la revista Le Débat, fue objeto de fuertes discusiones que permitieron dilucidar el rol jugado por la prensa y acceder al impensado evocado más arriba.

Así, se ve que, además de la dimensión jurídica, lo que se pone en tela de juicio es la relación con la Historia y con la construcción de esta última, más que la relación con los historiadores. Sin embargo los dos aspectos no se pueden disociar. La prensa se había centrado en la intervención de los historiadores que avalaban un compromiso en una cierta concepción de la responsabilidad de Vichy en el judeocidio. Ahora bien, en las revistas especializadas, los trabajos de los historiadores fueron utilizados para favorecer una concepción del Político y de una política memorial diferentes, a lo que se opuso Nicolas Weill, más fuertemente que en su propio diario (Le Monde), acusando a los historiadores que distinguen entre el antisemitismo de Vichy o de la Iglesia y el de los nazis, de exonerar a Vichy de su estatus de "co-autor" del Genocidio. Según él, el proceso aportó algo más que el juicio Eichmann a la comprensión del "crimen de escritorio", principalmente porque Maurice Papon no era antisemita. El se rebelaba contra el hecho que ciertos críticos se concentraban en dificultades que estarían relacionadas con las "metamorfosis del contexto político-memorial" y no con la naturaleza misma de este tipo de criminalidad (Weill, 1999: 108). Una crítica que, anclada en el escenario de la Política, está determinada también por el lugar social de los periodistas y de los historiadores. 


\section{Conclusión}

El análisis de la mediatización del testimonio-experto durante el proceso Papon, que integra al análisis de discurso teorizaciones sociológicas y epistemológicas, muestra que si bien existe acuerdo sobre la necesidad de una refundación política a partir de la Historia, las soluciones propuestas son antagonistas. Evidentemente, ellas no dependen únicamente de los servidores de Clio, aun cuando ellos son expertos o especialistas del período considerando que se desarrolla en varios escenarios, sino de un amplio conjunto de actores sociales, entre los cuales están los medios -de todo tipo- que contribuyeron a que surgieran las posiciones o a darles forma en el espacio público. Sobre este punto la diferencia entre prensa generalista y revistas especializadas se debe tanto a la estructuración de los soportes como a la relación que cada una mantiene con la Historia y por lo tanto con la experticia que le concierne. De aquí el interés, en este caso y en otros quizás, de asociar la sociología de la experticia con la de las profesiones, no solamente para adaptar las herramientas de análisis a los particularismos de los corpus, sino también para considerar la complejidad entre los lugares de pertinencia del discurso mediático. Como resultado del estudio, el conjunto teórico nos permitió concluir que, para los periodistas, se trató, durante el proceso, de actuar sobre la Historia para ajustarla a las expectativas del presente. Al contrario, para los científicos -sobre todo para aquellos que no prestaron testimonio- se trataba de conocerla mejor para comprender lo que representa actualmente, aun si con esto se facilita, por su discreción, la legitimidad de su actividad en tensión con la de otro grupo profesional muy cercano al de ellos.

\section{$-\quad$ Referencias bibliográficas}

Amiraux V. y Cefaï D. (2002). «Les risques du métier. Engagements problématiques en sciences sociales». Cultures \& Conflits. Obtenido desde http:// conflits.revues.org.

Baruch M. O. (1997). Servir l'État français. L’administration en France de 1940 à 1944. París: Fayard.

Bucher R. y Strauss A. L. (1961). «La dynamique des professions». En: La trame de la négociation. Sociologie qualitative et interactionnisme. París: L'Harmattan, pp. 67-86.

Burrin Ph. (1998, juin). «Vichy: quoi de neuf?». L’Histoire. No 222, pp. 80-81.

Charaudeau P. (1997). Le discours d'information médiatique, la construction du miroir social. Paris: INANathan.

(2001). «La télévision et l'autre-étranger. Conditions d'une étude». En: La télévision et la guerre. Déformation ou construction de la réalité? Le conflit en Bosnie (1990-1994). Bruxelles: De Boeck Université-INA, pp. 7-29.

Conan É. (1998). Le procès Papon. Un journal d’audience. París: Gallimard.

Conan É., Rousso H. (1994). Vichy, un passé qui ne passe pas. París: Gallimard (ed.: 1996)

Damamme D. y Lavabre M.-C. (2000). «Les historiens dans l'espace public». Sociétés contemporaines. No 39, pp. 5-21. 
Droit et société (1998). "Vérité historique, vérité judiciaire”. № 38.

Dumay J.-M. (1998). Le procès de Maurice Papon. La chronique de Jean-Michel Dumay. París: Fayard.

Fleury-Vilatte B. y Walter J. (2003). «Le procès Papon: de la médiatisation du discours historien à la controverse historiographique». Actes du colloque Supports, dispositifs et discours médiatiques à l'heure de l’internationalisation. Bucarest, 28 juin-2 juil.: Éd. de l'université de Bucarest. (2004). «Le procès Papon et les figures de l'historien-expert». En: La médiatisation de l'Histoire. De l'utilisation du passé dans la construction de l'actualité. Bruxelles: Bruylant.

Heinich N. (2002). «Pour une neutralité engagée». Questions de communication. No 2, pp. 117-127.

Jean J.-P., Salas D., dirs. (2002). Barbie, Touvier, Papon. Des procès pour la mémoire. París: Éd. Autrement.

L'Histoire (1998, juin). «Papon: leçons d'un procès». № 222.

Le Débat (1998, nov-dec). «Vérité judiciaire, vérité historique». No 102.

Ribémont Th. (2001, mai). «Le procès Papon: un cas d'expertise historienne?». Cahiers politiques, pp. 73-94.

Rousso H. (1998). «La hantise du passé». Entretien avec Ph. Petit. París: Textuel.

_ (2002). «L'expertise des historiens dans les procès pour crime contre l'humanité». En: Barbie, Touvier, Papon. Des procès pour la mémoire. París: Autrement, pp. 58-70.

Trépos J.-Y. (1996). La sociologie de l'expertise. París: Presses Universitaires de France.

(2002). «Lexpertise commeéquipement politique dela société civile».Questions de communication. No 2,pp. 7-18.

Walter J. (1995). Directeur de la communication. Les avatars d’un modèle professionnel. París: L'Harmattan. (2003). «Cadres du témoignage historique et médiatique, frontières disciplinaires». Questions de communication. No 3, pp. 11-30.

Weill N. (1999). «Penser le procès Papon».Le Débat. No 103, pp. 100-110.

Weill N. y Wieviorka A. (1994). «La construction de la mémoire de la Shoah: les cas français et israélien». Les Cahiers de la Shoah. No 1, pp. 163-191. 